\title{
Effects and clinical feasibility of a behavioral treatment for sleep problems in adult attention deficit hyperactivity disorder (ADHD): a pragmatic within-group pilot evaluation
}

Susanna Jernelöv ${ }^{1,2^{*}} \mathbb{D}$, Ylva Larsson ${ }^{1}$, Milagros Llenas ${ }^{1}$, Berkeh Nasri ${ }^{2}$ and Viktor Kaldo ${ }^{2,3}$

\begin{abstract}
Background: Sleep disturbances, including insomnia, are common in adult Attention Deficit Hyperactivity Disorder (ADHD). Treatment of choice for insomnia is cognitive behavioral therapy (CBT-i), but evidence is lacking for CBT-i in patients with ADHD.

The purpose of this study was to investigate if patients with insomnia and other sleep problems, at a specialist clinic for ADHD, benefit from a group delivered behavioral treatment based on CBT-i; whether insomnia severity improves following this treatment.
\end{abstract}

Methods: This pragmatic within-group pilot study with a pre to post and three-month follow-up design was set at a specialist psychiatric out-patient clinic for adult ADHD.

As an adjunct to care-as-usual at the clinic, a CBT-i-based group treatment targeting several sleep problems prevalent in the ADHD-population, was offered as 10 weekly 90-min group sessions and scheduled telephone support.

All outcome measures were subjectively reported by participants. Data analyzed with dependent t-tests according to intent-to-treat.

Results: Nineteen patients (37 [SD 13.7] years; 68\% female) with ADHD and subjectively reported sleep problems provided informed consent and pre-treatment measures. Patients had suffered from sleep problems for 15.3 [SD 13.4] years, 42\% used sleep medications, 79\% used stimulant medication(s).

At post-treatment, insomnia severity (Insomnia Severity Index; score range 0-28) had improved with 4.5 points (95\% $\mathrm{Cl}, 2.06-6.99, p=.002)$, at 3 months with 6.8 points $(95 \% \mathrm{Cl}, 4.71-8.91, p<.0001)$ from pre-treatment.

Conclusions: CBT-i adjusted for ADHD is promising for improving insomnia severity in adult patients at specialist psychiatric out-patient clinics, who suffer from ADHD and sleep disturbances.

Trial registration: Study registered with the Regional ethical review board in Stockholm, January 13th 2016, Study id: 2015/2078-31/1. Study registered retrospectively with Clinicaltrials.org, February 21st 2019, ID: NCT03852966.

Keywords: ADHD, Sleep problems, Behavioral treatment, Pilot evaluation

\footnotetext{
* Correspondence: susanna.jernelov@ki.se

'Division of Psychology, Department of Clinical Neuroscience, Karolinska Institutet, Stockholm, Sweden

${ }^{2}$ Centre for Psychiatry Research, Department of Clinical Neuroscience, Karolinska Institutet, \& Stockholm Health Care Services, Stockholm County Council, Stockholm, Sweden

Full list of author information is available at the end of the article
}

(c) The Author(s). 2019 Open Access This article is distributed under the terms of the Creative Commons Attribution 4.0 International License (http://creativecommons.org/licenses/by/4.0/), which permits unrestricted use, distribution, and reproduction in any medium, provided you give appropriate credit to the original author(s) and the source, provide a link to the Creative Commons license, and indicate if changes were made. The Creative Commons Public Domain Dedication waiver (http://creativecommons.org/publicdomain/zero/1.0/) applies to the data made available in this article, unless otherwise stated. 


\section{Background}

Attention Deficit Hyperactivity Disorder (ADHD) is a psychiatric disorder characterized by decreased attention, increased impulsivity and hyperactivity, and is often accompanied by deficits in vigilance and executive functioning [1]. In adults, a prevalence of ADHD of $2.5 \%$ has been reported [2]. ADHD in adults, as in children, is commonly treated with stimulant medication, but psychosocial interventions are also recommended as symptoms and impaired everyday functioning commonly persist even after pharmacological treatment [3]. As a consequence, psychological treatments to ameliorate ADHD problem areas are being developed and tested $[4,5]$.

Patients with ADHD often have several comorbid conditions [6], including sleep-wake problems. Adults with ADHD report more sleep problems than healthy controls $[7,8]$. One study found that $82.6 \%$ of patients with ADHD had ever had sleep problems, compared to $36.5 \%$ of controls [8], and not feeling refreshed in the morning is strongly associated with ADHD [9]. Insomnia, i.e. difficulties initiating or maintaining sleep and experiencing sleep-related daytime symptoms, is highly prevalent in this patient group [10, 11]. Delayed sleep phase syndrome, i.e. a delayed circadian rhythm, is also common [12-14], and can cause difficulties with both falling asleep and waking up early. These difficulties may be due to an altered endogenous rhythm, but may also be maintained by late habits due to executive difficulties.

Despite sleep problems such as insomnia and delayed sleep phase being prevalent in adults with ADHD, very few empirical studies specifically address their treatment in this patient group. For children with ADHD, several treatments have been tried in scientific evaluations. For instance, the use of melatonin to treat sleep onset insomnia and delayed sleep phase is fairly well established $[15,16]$. Unfortunately, studies on the efficacy of melatonin to treat insomnia in adult populations with ADHD are mostly lacking, but a few studies have investigated the use of light therapy with promising results $[17,18]$. Even though pharmacological treatment for insomnia is commonly used, hypnotics often do not result in satisfactory levels of symptom reduction or improvement in functioning in insomnia patients [19], and the few available studies on adult ADHD are no exception [20]. Given the less than optimal effects of pharmacological treatments and the risk of adverse interactions with prescribed medications, non-pharmacological interventions may be an attractive option for the management of insomnia in patients with ADHD.

Many factors may be involved in the development of insomnia, but maintaining factors are most often behavioral [21]. Perhaps then it is not surprising that insomnia can be successfully treated with specific behavioral interventions, as has been demonstrated previously $[22,23]$. In fact, cognitive behavioral therapy for insomnia (CBT-i) is now generally recommended as first line treatment, (e.g. [24-27]), and has also been shown to be effective when insomnia is comorbid with other problems, including several psychiatric problems [28, 29].

Interestingly, studies have shown that psychiatric symptoms may also improve with successful treatment of insomnia (e.g. [23, 30]). However, deteriorations have also been seen (e.g. [31]), and it is therefore important to investigate whether changes in core symptoms of ADHD may be seen after treatment of disturbed sleep.

Since sleep problems in ADHD are varied and complex, a sleep-focused CBT protocol should ideally target not only insomnia disorder according to DSM 5, but also other ADHD related sleep difficulties, such as delayed sleep phase and insufficient sleep hygiene. To our knowledge, however, no version of CBT-i has been tested in adults with ADHD as of yet.

\section{Aims of the study}

The aim of this paper is to perform a preliminary evaluation, to investigate if insomnia severity and ADHD symptoms improve following a novel group intervention based on CBT for insomnia and adjusted for patients with ADHD (CBT-i/ADHD), and to gauge if the treatment is feasible in a clinical setting.

\section{Methods \\ Design}

This is a pragmatic within-group (i.e. non-controlled) pilot study with a pre to post design and three-month followup. The study was conducted in collaboration with Northern Stockholm Psychiatry, Stockholm County Council, Sweden, and set at a specialist psychiatric out-patient clinic for adults with ADHD.

The authors assert that all procedures contributing to this work comply with the ethical standards of the relevant national and institutional committees on human experimentation and with the Helsinki Declaration of 1975, as revised in 2008. The study protocol was registered and approved by the regional ethical review board in Stockholm, Sweden (Study id: 2015/ 2078-31/1), and written informed consent was obtained from all participants.

\section{Participants}

The study included 19 patients at a specialist psychiatric out-patient clinic for adult ADHD. Apart from having an ADHD diagnosis and being a registered patient at the clinic, inclusion in this study required self-reported sleep problems, being interested and 
able to participate in the group treatment, and to return pre-treatment study questionnaires including a consent form before deadline. Medication use or comorbidities were thus not cause for exclusion, as long as the patient was able to participate in the group treatment. Patients were recruited during the fall of 2015 and spring of 2016 through referral from clinic staff, and the experimental behavioral treatment was an adjunct to care-as-usual at the clinic.

For participant flow through study, see Fig. 1.

\section{Procedure and assessments}

Interested participants received a telephone call from study staff, with information about the study and the treatment. Patients who were still interested in participating were sent pre-treatment questionnaires to be returned before treatment start. Demographic data and other patient characteristics including diagnoses and medication use were collected at pre-treatment, also using self-report questionnaires. Outcome measures were self-rated and administered at pre-treatment, post-

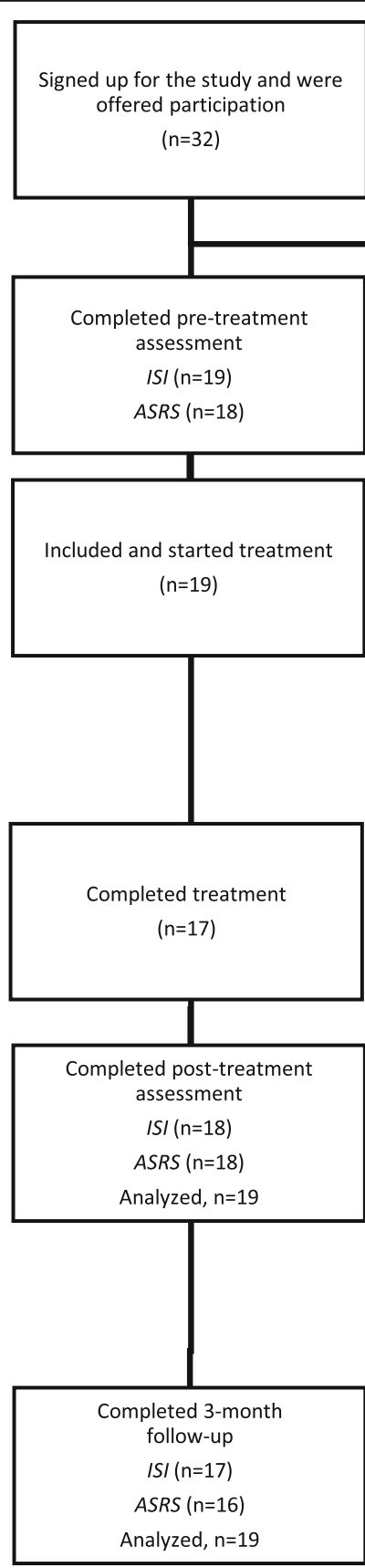

Declined treatment $(n=7)$

Did not complete ISI pre-treatment assessment $(n=3)$

Did not give consent $(n=3)$ 
treatment and three-month follow-up. Treatment evaluation forms were handed out after the last treatment session and patients were asked to fill them out and return them before leaving.

\section{Screening and background}

The SLEEP-50 [32] was used to screen for sleep disorders. Additional background information was collected using a form with questions on age, sex, marital status, education level, and current occupation and questions on psychiatric diagnoses and problems, medication use, and morning-eveningness. More specifically, for type of ADHD, patients were asked to choose which described their type best: Predominantly inattentive, Predominantly hyperactive/ impulsive, Combined subtype, or NOS; for psychiatric diagnoses an open-ended question was used; for psychiatric problems during the last year, patients were given a list and asked to mark which applied to them; medication use was gauged with an open ended questions with examples, and medications were classified according to stated use and/or substance; circadian type was measured with the question on morning-eveningness from the Karolinska Sleep Questionnaire, on a five point scale (Extreme morning type, morning type, intermediate type, evening type, extreme evening type) [33].

\section{Primary symptom outcome}

To evaluate insomnia severity, the Insomnia Severity Index (ISI) was used. This 7-item patient-reported questionnaire has adequate psychometric properties and is sensitive to change [34]. A 5-point scale (0-4) is used to rate each item, yielding a total score of 0 to 28 . In this study, the Swedish adolescent version of the ISI (ISI-a [35]) was used. The scale has shown adequate internal reliability (Cronbach's alpha $=.83$ ) and correspondence with other measures of insomnia [35]. Levels of insomnia severity were defined according to Bastien et al. [34]: No clinically significant insomnia (i.e. in remission): $0-7$ points; Subthreshold insomnia: $8-14$ points; Clinical Insomnia, moderate severity: 15-21 points; Clinical insomnia, severe: $22-28$ points.

\section{Secondary symptom outcome}

Severity of ADHD-symptoms was measured with the Adult ADHD Self-Report Scale (ASRS), consisting of 18 items related to ADHD-symptoms (according to the DSM-IV), to be rated on a 0 (never) to 4 (very often) scale, yielding a score ranging from 0 to 72 points. The scale has two subscales for inattention and hyperactivity symptoms respectively, each 0 to 36 points. Cut off for probable ADHD is 17 points and for highly probable ADHD 24 points, on either of the subscales. The scale has shown good internal reliability (Cronbach's alpha $=.88$ ) and good construct validity (i.e. high correlation with clinicians' assessments of ADHD-symptoms) [36].

\section{Patient compliance and satisfaction with treatment}

Patient compliance was measured with (a) proportion of patients dropping out of treatment and (b) average number of group sessions attended.

Patient satisfaction with treatment was measured with a treatment evaluation questionnaire constructed for the study asking (c) if the patient would recommend this type of treatment to a friend with ADHD and sleep difficulties, and (d) the patient's overall impression of the treatment (1-6, where 1 represents "very poor", 2 "poor", 3 "quite poor", 4 "quite good", 5 "good", 6 "very good"). Patients were also asked about their opinion on (e) most important treatment components.

\section{Treatment}

The treatment consisted of weekly 90-min sessions over the course of 10 weeks, and was based on CBT-i, with adjustments to both content and format. Content was adjusted by adding behavioral components targeting other sleep and circadian problems common in this patient group, such as delayed sleep phase and insufficient sleep hygiene, and by adding components targeting commonly occurring difficulties with planning and organizing, to help patients succeed in applying the sleep related treatment components (e.g. by using an alarm and a calendar, as suggested by Safren et al. [37]). Format was adjusted for instance by shortening sessions, lengthening treatment period and including a short (5-20 min) scheduled telephone call from one of the group leaders between sessions. All adjustments were made to better suit the needs of the patient group with ADHD. Regular CBT-i-components were sleep scheduling with the option of sleep compression based on sleep diary data, stimulus control, relaxation/visualization and cognitive restructuring. To work behaviorally with sleep phase problems, circadian nadir was approximated using a series of questions regarding patients' circadian habits, for instance preferred and spontaneous rise-time, and most difficult time staying awake during the night, in order to find each patients optimal time-point for natural light exposure, meal-times and activity scheduling. For an overview of session content, please see Table 1.

Groups were closed, i.e. group members were the same from start to finish. Each group had between 6 and 10 participants and was led by two therapists. All therapists had undergone extensive CBT-training and training in the current treatment manual, and received 
Table 1 Session content

\begin{tabular}{|c|c|c|}
\hline Session & Contents & Homework (use of strategies) \\
\hline 1 & $\begin{array}{l}\text { Introduction to treatment, information about } \\
\text { CBT and CBT-i models of treatment. Introduction } \\
\text { to the use of organizing-strategies. Discussing } \\
\text { treatment goals. }\end{array}$ & $\begin{array}{l}\text { Fill out a sleep diary every day. Use calendar, to-do list, } \\
\text { distractibility reduction skills, set treatment goals. }\end{array}$ \\
\hline 2 & $\begin{array}{l}\text { Problem-solving and behavioral experiments if } \\
\text { needed }{ }^{a} \text {. Psychoeducation about sleep, sleep } \\
\text { myths, sleep and ADHD, and effects of ADHD } \\
\text { medication on sleep. Role of relaxation and use of relaxation techniques. }\end{array}$ & $\begin{array}{l}\text { If needed, discuss ADHD-medication timing with prescribing } \\
\text { psychiatrist. Stabilizing sleep medication. Practicing relaxation } \\
\text { techniques to be used both during daytime and in bed. }\end{array}$ \\
\hline 3 & $\begin{array}{l}\text { Setting the circadian rhythm. Approximating } \\
\text { the circadian nadir of each patient and setting } \\
\text { the appropriate "light schedule". b }\end{array}$ & $\begin{array}{l}\text { Use light and darkness systematically, according to the light } \\
\text { schedule developed in session. }\end{array}$ \\
\hline 4 & $\begin{array}{l}\text { Regularizing sleep schedule and adjusting other } \\
\text { activities accordingly, to help set circadian rhythm } \\
\text { and use sleep pressure to improve sleep, use of a } \\
\text { morning-routine to get up in time. Develop each } \\
\text { patient's individual sleep schedule. }\end{array}$ & $\begin{array}{l}\text { Follow the sleep schedule developed in session (after the first } \\
\text { routine to get up in the morning. }\end{array}$ \\
\hline 5 & $\begin{array}{l}\text { In the evening: activity level, routines and } \\
\text { management of pre-sleep worry. Identifying } \\
\text { individual needs and planning how to work with them. }\end{array}$ & $\begin{array}{l}\text { Gradually-less arousing activities } 1,5-2 \mathrm{~h} \text { before bedtime, } \\
\text { "worry time" if needed, use a simple evening routine. }\end{array}$ \\
\hline 6 & $\begin{array}{l}\text { Follow up on treatment progress and goals. Stimulus } \\
\text { control and sleep hygiene. Identifying individual sleep } \\
\text { hygiene needs and planning how to work with it. }\end{array}$ & $\begin{array}{l}\text { Getting out of bed when unable to sleep for } 20 \mathrm{~min} \text {. Follow } \\
\text { individual plan for sleep hygiene practices. }\end{array}$ \\
\hline 7 & $\begin{array}{l}\text { Daytime activity, variability and pacing. Identifying } \\
\text { individual needs and planning the work. Non } \\
\text { sleep-disturbing ways to handle fatigue. }\end{array}$ & $\begin{array}{l}\text { Physical activity. Increase variability of activity level, use non } \\
\text { sleep-disturbing ways to handle fatigue. }\end{array}$ \\
\hline 8 & Cognitive activity and sleep. Cognitive restructuring. & Identify and manage sleep disturbing thoughts. \\
\hline 9 & $\begin{array}{l}\text { Acceptance and mindfulness. Summarize the treatment, } \\
\text { choose a strategy to apply during the last week. }\end{array}$ & $\begin{array}{l}\text { Work with the chosen strategy, and try acceptance and } \\
\text { mindfulness strategies. }\end{array}$ \\
\hline 10 & $\begin{array}{l}\text { Evaluation of treatment goals. Relapse prevention. Create } \\
\text { an individual Sleep Plan based on strategies from the treatment. }\end{array}$ & Follow the Sleep Plan, including relapse prevention. \\
\hline
\end{tabular}

a Problem-solving and behavioral experiments applied each following session, if needed

${ }^{\mathrm{b}}$ Continued work with both light and sleep schedule carried on to all subsequent sessions

weekly supervision by an experienced clinician and behavioral sleep medicine expert (SJ).

\section{Power considerations}

The expected within-group effect size for the ISI was Cohen's $d=1.0$, based on previous studies of insomnia treatment in comorbid patient groups, (e $\mathrm{g}$ $[23,38,39])$. To achieve a power of $80 \%$, with an alpha level of $.05,16$ participants were needed. Since attrition was expected we aimed for 20 included participants.

\section{Data analysis}

For outcome measures (ISI and ASRS), imputation for missing items in questionnaires was performed using the Corrected Item Mean substitution [40]. Data was screened for normality, and as none of the variables were statistically non-normally distributed at any of the time-points (Kolmogorov-Smirnov p's $.108<.200$ ), data from missing questionnaires at the post-treatment and follow-up assessments were imputed using the multiple imputation procedure in
SPSS 24. Missing data from the qualitative evaluation questionnaires were not imputed. Dependent samples $t$-tests were performed to assess whether changes from pre- to post-treatment and from pretreatment to follow-up were statistically significant. Dependent t-tests comparing post-treatment to follow-up scores were also performed to test maintenance of improvements. The effect sizes of withingroup changes between the measurement points were calculated as Cohen's $d$, including 95\% confidence intervals [41]. Means, standard deviations, and standard errors of t-tests and effect sizes were pooled from five imputations using "Rubin's rules" [42] and the small sample correction for pooled degrees of freedom [43]. To test for differences in insomnia symptom severity level (remission, subthreshold, moderate or severe) at pre-treatment, post-treatment and follow-up assessments, the Wilcoxon signed ranks test was performed, with missing data replaced by last observation carried forward. Treatment evaluation forms included only single item questions and qualitative data, and data from these forms is reported descriptively only. 


\section{Results}

As can be seen in Table 2, participants were mainly female, reported on average 3.3 current comorbid psychiatric problems (in addition to ADHD and sleep problems), a majority used stimulant medication(s) for their ADHD, all patients screened positive for insomnia, a majority also screened positive for one or more additional sleep disorders including sleep phase problems, and about two in five used sleep medications.

\section{Data attrition}

The ISI was completed by 18 (95\%) of the 19 participants at post-treatment and $17(89 \%)$ at 3-month follow-up assessments. The corresponding numbers for ASRS was 18 (95\%) and 16 (84\%). Missing items for these questionnaires were less than $1 \%(0.4-0.9)$ at each assessment point.

Treatment evaluation forms were handed in by 13 patients. Missing items were more common in these questionnaires, i.e. $7 \%$.

\section{Symptom related outcomes}

Means, standard deviations, and within group effect sizes, including confidence intervals, for symptom related outcomes at all assessment points are reported in Table 3.

For insomnia severity, intent-to-treat analyses with dependent t-tests showed statistically significant improvements on the ISI from pre- to post-treatment ( $\mathrm{t}(13.63)=3.96, p=.002)$, from pre-treatment to follow-up ( $\mathrm{t}(13.47)=7.01, p<.0001)$, but not from post-treatment to follow-up ( $\mathrm{t}(12.74)=1.73, p=.11$ ). On the ISI, the within-group effect size from pretreatment to post-treatment was $d=0.84$, and from pre-treatment to follow-up a larger $d=1.52$.

Two sensitivity analyses were performed. The first was conducted using non-parametric (the Wilcoxon signed ranks test) as opposed to parametric tests, and this analysis was conducted using non-imputed data (i.e. also testing for possible problems due to imputations). This analysis confirmed and somewhat extended the results from the t-tests, indicating that not only were the median ranks for the post-treatment assessment statistically significantly lower than the median ranks for pre-treatment assessment, but also that the median ranks for the follow-up assessment were statistically significantly lower than the median ranks for the post-treatment assessment ( $p$ 's $.031>.000$ ), thus indicating continued improvement between post-treatment and follow-up. These results then support the initial findings, using non-parametric analyses and non-imputed data. The second sensitivity analysis was conducted to control for possible social desirability in the reporting of
Table 2 Participant demographics, clinical profile and medication use at pre-treatment

\begin{tabular}{|c|c|}
\hline Variable & Total $n=19$ \\
\hline \multicolumn{2}{|l|}{ Age } \\
\hline Mean (Range) & $37.0(19-57)$ \\
\hline \multicolumn{2}{|l|}{ Sex } \\
\hline Female & $13(68 \%)$ \\
\hline \multicolumn{2}{|l|}{ Marital Status } \\
\hline Single & $9(47.4 \%)$ \\
\hline $\begin{array}{l}\text { Married/registered partnership/in a } \\
\text { relationship }\end{array}$ & $8(42.1 \%)$ \\
\hline Divorced/widow/widower & $1(5.3 \%)$ \\
\hline Other & $1(5.3 \%)$ \\
\hline \multicolumn{2}{|l|}{ Educational Level } \\
\hline Primary school & $1(5.3 \%)$ \\
\hline Secondary school & $9(47.4 \%)$ \\
\hline University & $9(47.4 \%)$ \\
\hline \multicolumn{2}{|l|}{ Occupation } \\
\hline Working/studying/self-employed & $9(47.4 \%)$ \\
\hline On sick leave, disability pension etc & $8(42.1 \%)$ \\
\hline Other & $2(10.5 \%)$ \\
\hline \multicolumn{2}{|l|}{ Pre-treatment ADHD severity ${ }^{a}$} \\
\hline Overall, Mean (SD) & $43(13.6)$ \\
\hline Inattention subscale, Mean (SD) & $24(7.2)$ \\
\hline Hyperactivity/impulsivity, Mean (SD) & $20(7.9)$ \\
\hline \multicolumn{2}{|l|}{ ADHD-subtype } \\
\hline Predominantly hyperactive/impulsive & $4(21 \%)$ \\
\hline Predominantly inattentive & $6(32 \%)$ \\
\hline Combined subtype & $7(42 \%)$ \\
\hline NOS & $1(5 \%)$ \\
\hline Current comorbid psychiatric symptoms & $13(68 \%)$ \\
\hline \multicolumn{2}{|l|}{ Comorbid psychiatric problems in the past year ${ }^{\mathrm{e}}$} \\
\hline Depression & $15(79 \%)$ \\
\hline Anxiety & $13(68 \%)$ \\
\hline Panic attacks & $8(42 \%)$ \\
\hline Excessive worry & $8(42 \%)$ \\
\hline Post-traumatic stress & $4(21 \%)$ \\
\hline Specific phobia & $4(21 \%)$ \\
\hline Intrusive thoughts and/or impulses & $4(21 \%)$ \\
\hline Psychosis & $1(5 \%)$ \\
\hline Manic episodes & $1(5 \%)$ \\
\hline Alcohol and/or substance abuse/addiction & $1(5 \%)$ \\
\hline Other & $3(16 \%)$ \\
\hline \multicolumn{2}{|c|}{ Number of comorbid psychiatric problems in the past year } \\
\hline Mean (SD) & $3.3(2.6)$ \\
\hline \multicolumn{2}{|l|}{ Sleep Disorder Screening ${ }^{b}$} \\
\hline Insomnia & $19(100 \%)$ \\
\hline
\end{tabular}


Table 2 Participant demographics, clinical profile and medication use at pre-treatment (Continued)

\begin{tabular}{|c|c|}
\hline Variable & Total $n=19$ \\
\hline Nightmares & $15(79 \%)$ \\
\hline Circadian rhythm sleep disorder & $14(73 \%)$ \\
\hline Sleep apnea & $14(73 \%)$ \\
\hline Restless legs/Periodic limb movements disorder & $10(53 \%)$ \\
\hline Narcolepsy & $4(21 \%)$ \\
\hline $\begin{array}{l}\text { Daytime impairment (irritation, concentration } \\
\text { difficulties etc.) }\end{array}$ & $18(95 \%)$ \\
\hline \multicolumn{2}{|l|}{ Years with Sleep Problems ${ }^{f}$} \\
\hline Mean (range) & $15.3(1.5-40)$ \\
\hline \multicolumn{2}{|l|}{ Pre-treatment Insomnia severity ${ }^{c}$} \\
\hline Mean ISI score (SD) & $15(3.9)$ \\
\hline Clinical insomnia, severe (22-28 points) & $1(5 \%)$ \\
\hline Clinical insomnia, moderate severity (15-21 points) & $11(58 \%)$ \\
\hline Subthreshold insomnia (8-14 points) & $6(32 \%)$ \\
\hline No clinically significant insomnia (0-7 points) & $1(5 \%)$ \\
\hline \multicolumn{2}{|l|}{ Circadian type $\mathrm{dg}^{\mathrm{d}}$} \\
\hline Extreme morning type & $1(5 \%)$ \\
\hline Morning-type & $0(0 \%)$ \\
\hline Intermediate type & $2(11 \%)$ \\
\hline Evening-type & $3(16 \%)$ \\
\hline Extreme evening-type & $12(63 \%)$ \\
\hline Current stimulant use, n (\%) & $15(79 \%)$ \\
\hline \multicolumn{2}{|l|}{ Stimulants ${ }^{\mathrm{e}}$} \\
\hline Methylphenidate & $7(37 \%)$ \\
\hline Dextroamphetamine & $4(21 \%)$ \\
\hline Lisdexamfetamine & 7 (37\%) \\
\hline Current sleep medication use, n (\%) & $8(42 \%)$ \\
\hline \multicolumn{2}{|l|}{ Sleep medications ${ }^{\mathrm{e}}$} \\
\hline Melatonin & $4(22 \%)$ \\
\hline Z-drugs & $2(11 \%)$ \\
\hline Antipsychotics & $3(16 \%)$ \\
\hline Antihistamines & $2(11 \%)$ \\
\hline Current use of other medication, $\mathrm{n}(\%)$ & $11(58 \%)$ \\
\hline \multicolumn{2}{|l|}{ Other medications ${ }^{e}$} \\
\hline Anticonvulsant & $3(16 \%)$ \\
\hline Antidepressant & 7 (37\%) \\
\hline Other & $8(42 \%)$ \\
\hline
\end{tabular}

${ }^{\text {aAdult ADHD Self-Report Scale [36] }}$

${ }^{\mathrm{b}}$ Sleep-50 [32]

Insomnia Severity Index [34]

${ }^{d}$ Morning-eveningness question from the Karolinska Sleep Questionnaire [32]

${ }^{\mathrm{e}}$ Each individual may mark more (or less) than one

${ }^{\mathrm{f}} n=12$

${ }^{g} n=18$ insomnia severity, using patient's score for their overall impression of the treatment as a proxy for social desirability and entered as covariate in an ANCOVA with ISI score at pre- post and follow-up as the dependent variable. This sensitivity analysis showed a statistically significant effect of time $(p=.001)$, but the interaction with patient's overall impression of the treatment was not statistically significant $(p=.673)$, indicating that the reporting of insomnia severity was not governed by patients' treatment satisfaction.

Figure 2 shows individual ISI scores at pre- post and follow-up assessments. As can be seen, three patients' insomnia severity did not improve during the treatment period. These three patients had all improved from post-treatment to follow-up assessments. From pre- to follow-up assessments, one patient $(5 \%)$ had a 4-point increase on the ISI, while at post-treatment assessment this patient had reported lower levels of insomnia.

Figure 3 shows the proportion of patients at different levels of insomnia severity over the measurement points, with last observation carried forward for the two patients with missing data described above. The results from the Wilcoxon signed ranks tests indicate that patients moved from a higher to a lower severity level, i.e. the median post-treatment ranks were statistically significantly lower than the pre-treatment ranks ( $M d n$ Pre $=3, M d n$ Post $=2, \mathrm{Z}=.000, p=.003$ ), and that the lower level was maintained at threemonth follow-up, i.e. median follow-up ranks were significantly lower than the pre-treatment ranks $(M d n$ Pre $=3, M d n$ FU $=2, \mathrm{Z}=8.000, p=.000)$, but did not differ from the post-treatment ranks $(M d n$ Post $=2, M d n \mathrm{FU}=2, \mathrm{Z}=15.000, p=.166$ ).

For ADHD-symptoms, intent-to-treat analyses with dependent $\mathrm{t}$-tests showed significant improvements on the ASRS from pre-treatment to three-month follow-up (t $(8.87)=2.77, p=.02, d=0.34)$ but not from pre- to post-treatment ( $\mathrm{t}(11.77)=1.38, p=.20)$ or from post-treatment to follow-up (t $(15.45)=1.00$, $p=.33)$. The within-group effect size from pre-treatment to follow-up was in the small range.

Post hoc analyses for ASRS subscales (see Table 3) showed statistically significant changes on the hyperactivity subscale between pre-treatment and post-treatment ( $\mathrm{t}(17)=2.15, p=.05, d=0.28)$, and between pretreatment and three-month follow-up assessments ( $\mathrm{t}$ $(17)=4.14, p=.001, d=0.44)$. At three-month follow-up, patient average on the hyperactivity subscale ended up just below the cut-off for probable ADHD-diagnosis (i.e. $<17)$. The inattentive subscale did not show a statistically significant change from pre-treatment to post-treatment assessment, but a statistically significant change 
Table 3 Means, standard deviations and effect sizes for symptom related outcomes, observed data with imputations, $\mathrm{n}=19$

\begin{tabular}{|c|c|c|c|c|c|c|c|c|c|}
\hline \multirow[t]{2}{*}{ Measure } & \multicolumn{2}{|c|}{ Pre-treatment } & \multicolumn{2}{|c|}{ Post-treatment } & \multicolumn{2}{|c|}{ Three-month Follow-up } & \multicolumn{3}{|c|}{ Within Group Effect Size $d(95 \%$ Cl) } \\
\hline & $M$ & SD & $M$ & SD & $\bar{M}$ & SD & Pre-post & Pre-Fu3 & Post-Fu3 \\
\hline$|S|$ & 15.4 & 4.2 & 10.9 & 6.0 & 8.6 & 4.7 & $0.84^{* * *}(0.31-1.37)$ & $1.52^{* * *}(0.87-2.18)$ & $0.42(-0.12-0.95)$ \\
\hline ASRS (total) & 43.2 & 13.2 & 40.2 & 15.7 & 38.7 & 13.0 & $0.20(-0,12-0.51)$ & $0.34^{*}(0.05-0.62)$ & $0.09(-0.10-0.29)$ \\
\hline Inattention & 23.7 & 7.2 & 22.2 & 8.1 & 21.6 & 6.7 & $0.19(-0.01-0.39)$ & $0.31^{*}(0.05-0.57)$ & $0.08(-0.18-0.34)$ \\
\hline Hyperactivity & 19.9 & 7.9 & 17.6 & 8.4 & 16.5 & 6.9 & $0.28^{*}(0.00-0.55)$ & $0.44 * * *(0.20-0.69)$ & $0.14(-0.07-0.35)$ \\
\hline
\end{tabular}

ISI insomnia severity index, ASRS adult ADHD self-report scale

${ }^{*} p<0.05,{ }^{* *} p<0.01,{ }^{* * *} p<0.001$

was seen between pre-treatment and three-month follow-up assessment ( $\mathrm{t}(17)=2.54, p=.02, d=0.31)$.

\section{Patient compliance and satisfaction with treatment}

a) Proportion of patients dropping out of treatment. Two patients (11\%) dropped out of treatment. One of these patients attended 1 session and the other 2 sessions before not showing up, and subsequently declining further participation, stating personal reasons for their discontinuation.

b) Average number of sessions attended. Patients attended an average of 7.2 out of 10 sessions (median $=8$ ). Patients who did not drop out of treatment attended an average of 7.9 sessions $($ median $=8)$. Most missed sessions were due to illness or more pressing engagements, such as work or doctor's appointments. Some patients had a hard time making it to the sessions due to difficulties planning and organizing. Patients who missed a session without a cancellation were telephoned by the therapist as soon as possible after the session, in addition to their regular telephone appointment.

c) Patients' satisfaction with treatment. All thirteen patients who completed the treatment evaluation form would recommend the intervention to a friend with ADHD and sleep problems. For the overall impression, twelve patients answered and six patients rated 6 (very good), five rated 5 (good), and one rated 4 (quite good).

d) Subjectively reported most important treatment components. 11 patients reported on most useful treatment components, and the three most commonly reported were: systematic use of light in the morning (reported by eight participants, 73\%) and darkness in the evening (eight participants, $73 \%$ ), and the use of a sleep schedule (seven participants, 64\%). Other treatment components that were mentioned were Bedtime routines, the $17+7$ rule, Relaxation, Stimulus Control, Morning routines, Psychoeducation about sleep, No napping, Engagement in physical or outdoor activity during the day, Worry time, Limit the use of tablets/

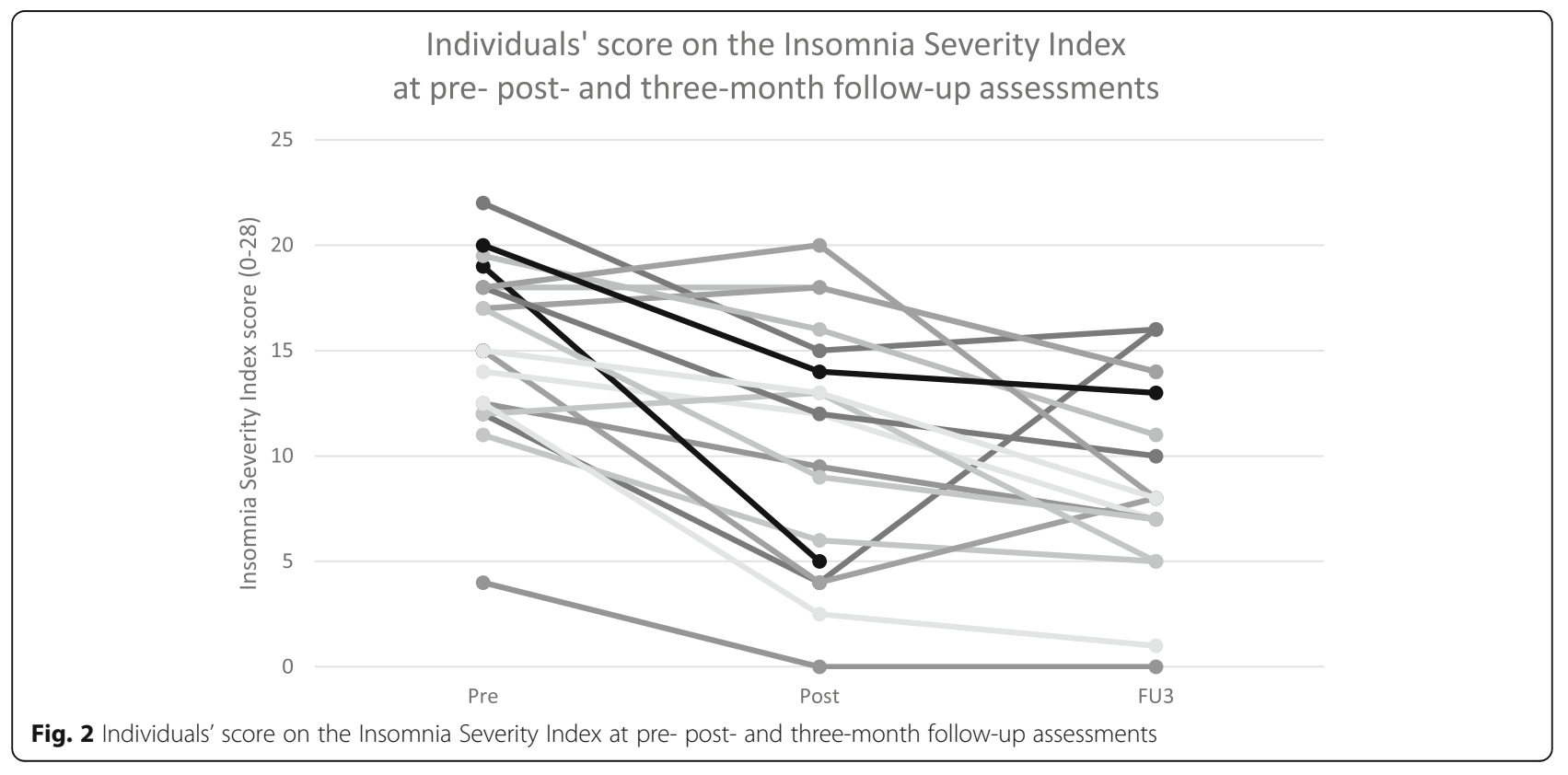




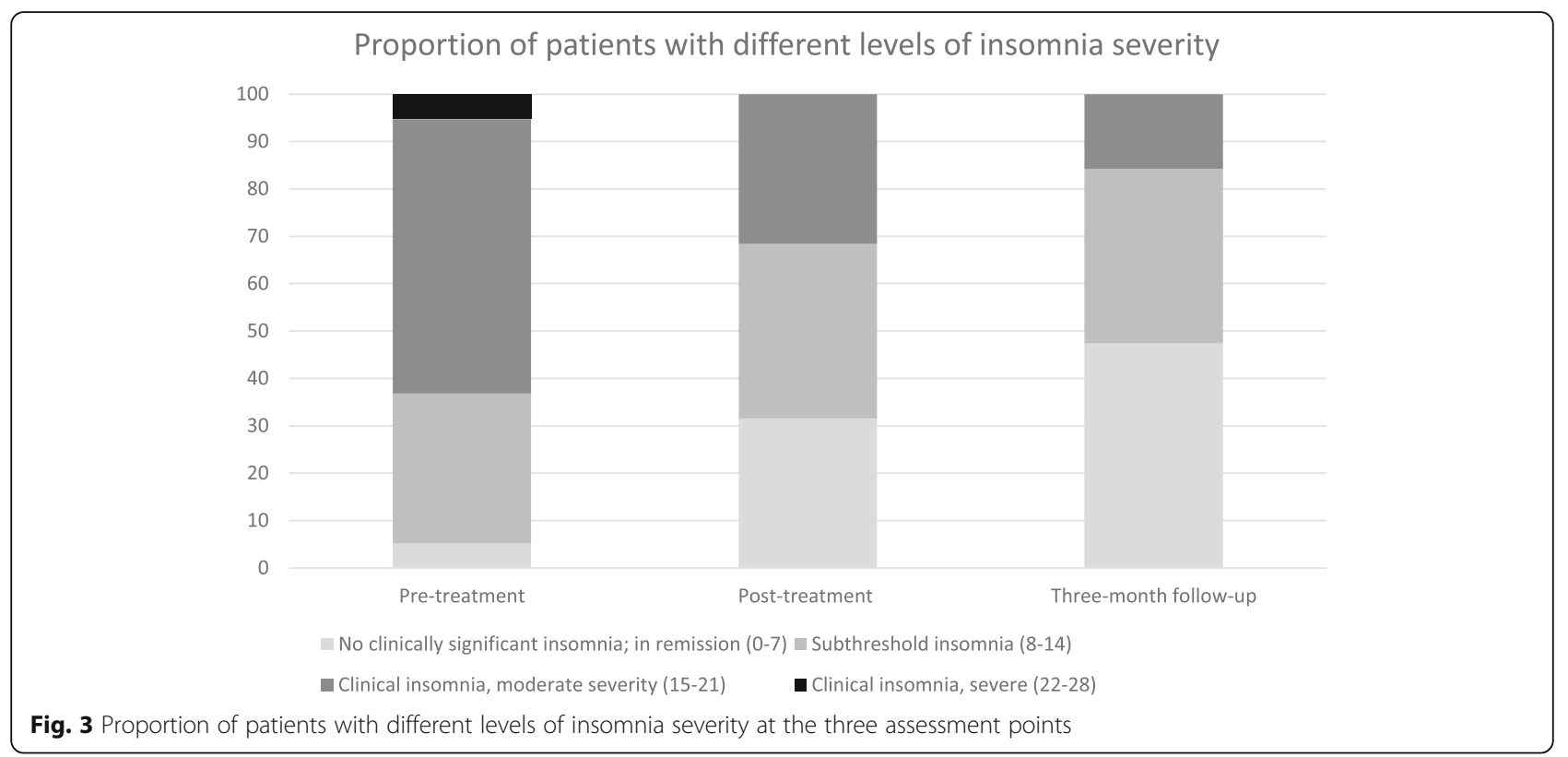

telephones in bed and other Sleep hygiene practices.

\section{Discussion}

In this pragmatic within-group pilot study, a novel group intervention based on CBT for insomnia, adjusted for patients with ADHD (CBT-i/ADHD), was investigated in a clinical setting. We conducted a preliminary evaluation of possible effects of the intervention, and gauged patient compliance and satisfaction, at a specialist psychiatric out-patient clinic. We found that insomnia severity improved significantly with a medium to large withingroup effect from pre-treatment to post-treatment assessments, and was stable at three-month follow-up. We also found an improvement in ADHD symptoms at three-month follow-up compared to pre-treatment. In addition, patient compliance with the intervention in a clinical setting was good, since most participants came to an adequate number of treatment sessions. Finally, patients were satisfied with the treatment. Overall, these results point to a promising treatment that is acceptable and feasible in a clinical setting.

The sleep related adjustments made to this treatment manual were made based on the literature on sleep problems in ADHD, and included components targeting insomnia and other sleep and circadian problems such as sleep phase shifts and problems with sleep hygiene and waking up in the morning. Quite recently, Harvey and Buysse suggested a new way of working with sleep problems in youth and potentially other patient groups, the so called TranS-C-approach $[44,45]$. Interestingly, the TranS-C approach includes many of the same strategies that were adopted in this treatment, including systematic work with light and other zeitgebers, and wake-up routines. To our knowledge, no evaluation of the TranS-C-approach has been published as of yet, but the Oxford Ward sLeep Solution (OWLS) for psychiatric in-patients [46] is building on similar ideas. A pilot trial of the OWLS was recently published, suggesting the OWLS would lead to improvement in insomnia severity, and fewer days in admission [47]. The OWLS is used in patients with different psychiatric problems, pointing to the possibility that other patient groups might also benefit from some of the adaptations used in the CBT-i/ ADHD. For instance, dysregulation of the circadian system has been implicated in both patients with bipolar disorder [48] and psychotic disorders [49] so these patient groups might also benefit from strategies aimed at stabilizing the circadian rhythm. However, although our treatment contains components aiming to shift and stabilize participants' circadian rhythm, we do not measure circadian rhythmicity, and thus do not know whether it changes following this treatment. Future studies are needed to investigate this aspect.

Other alterations were made to increase the likelihood for patients with ADHD to achieve the sleep related behavior changes that are central to this treatment (e.g. keeping constant bedtimes, timing light exposure, and doing relaxation exercises). To this end, we added strategies for organizing and planning in the first session, drawing from the Safren manual on CBT for ADHD [37]. We also added telephone calls from a therapist between each treatment session. Neither of these components is commonly included in a CBT-i-treatment. In addition, we increased the number of session and shortened the length of sessions. Which - if any - of these 
adaptations are important, we do not know. The present study was not designed to test the usefulness or relative importance of different treatment components, but that is an important area for future research studies.

The effects on insomnia severity in the present study are in the medium to large range (post-treatment, $d=$ 0.84 ; three-month follow-up, $d=1.52$ ), and the proportion of patients in remission increase over time $(32 \%$ at post-treatment, and $42 \%$ at three-month follow-up) while the proportion of patients with moderate or severe insomnia decreased over time $(63 \%$ at pre-treatment, $32 \%$ at post-treatment, and $16 \%$ at three-month followup). Considering patients have at least one other disabling psychiatric disorder (i.e. ADHD) and most have additional comorbidities, it is encouraging that patients, after this behavioral intervention, reported improvements in insomnia severity and proportion of remitters after treatment comparable to previous studies with non-ADHD populations [29, 38].

In addition to the improvement seen in insomnia severity, ADHD-symptoms improved slightly between pre-treatment and three-month follow-up assessments, which is especially interesting considering about $80 \%$ of patients already have pharmacological treatment for their ADHD. The improvement seen in this pilot is small in effect $(d=0.34)$, and due to the withingroup study design it cannot be causally attributed to the treatment, but to our knowledge this is the first study showing possible improvements on ADHD symptoms after a behavioral sleep intervention. Posthoc analyses indicated that the change was larger on the hyperactivity sub-scale $(d=0.44)$, and at threemonth follow-up patient average actually fell below the cut-off for probable ADHD on this sub-scale (i.e. 17 points). This is especially interesting since arousal is an important aspect of sleep, and included in several of the theoretical models of insomnia (e.g. the cognitive behavioral model of insomnia [50] and hyperarousal theory [51]). Inability to control arousal or wrongly timed arousal can cause acute insomnia, and can be a contributing factor for chronic sleep problems. In ADHD, hyperactivity is a core symptom which has been associated with insomnia [52]. The decrease in hyperactivity symptoms seen in the patients in this study could conceivably be indicative of an amelioration of negative consequences of poor sleep with symptoms similar to ADHD-symptoms, and/or a decrease in arousal, possibly associated with a shift in the circadian rhythm, following the treatment. These possible mechanisms are intriguing and warrant further study. Although the decrease in ADHD-symptoms is smaller than that produced by psychological treatments targeting ADHD-symptoms (e.g. $[4,5])$ it is nonetheless very encouraging.
Whether these results would hold in a randomized trial remains an empirical question. Since this is only a small pilot with no control group, results should be interpreted with caution. Apart from this basic methodological weakness, a couple of limitations should be noted; sleep problems are not diagnosed but only screened for, there is no objective measure of sleep and no sleep diary data; pharmacological interventions during the study period are not controlled for, neither for insomnia nor for ADHD, and we can thus not exclude the possibility that improvements are due to other factors, for instance changes in medication. Indeed, both stabilizing the use of sleep medications, and adjusting ADHD medication timing, was encouraged in this intervention. However, neither was reported by the participants as the most useful treatment component, indicating that other strategies were perceived by patients as having greater impact on their sleep.

Important strengths of this study are the low intervention drop-out and study attrition. Also, therapists, who were psychologist students with limited clinical experience, could successfully deliver this treatment, suggesting the treatment manual would be useful also to clinicians who are not experts in behavioral sleep medicine. Finally, the fact that the intervention was given in a naturalistic setting to patients at a specialist psychiatric out-patient clinic, makes direct generalization to this patient group possible.

To confirm and elaborate on these positive preliminary results, a randomized trial is currently being conducted at the Department of ADHD, Northern Stockholm Psychiatry (Stockholm, Sweden).

\section{Conclusions}

The CBT-i/ADHD is a newly developed behavioral treatment for patients with ADHD, insomnia and other sleep problems, based on CBT-i and with adjustments to account for problems specific to the ADHD-population. Insomnia severity was reduced with medium to large within-group effect size from pre-treatment assessment to post-treatment and follow-up assessments. A small improvement was also seen in ADHD-symptoms at three-month follow-up, and the treatment was feasible in clinical practice. However, since no control group was used, results should be interpreted with caution, and further studies will be needed to create an evidence base for the use of non-pharmacological alternatives to treat sleep problems, with the potential to greatly improve care and quality of life, in patients with ADHD.

\section{Abbreviations}

ADHD: Attention deficit hyperactivity disorder; ASRS: Adult ADHD self-report scale; CBT: Cognitive behavioral therapy; CBT-i: Cognitive behavioral therapy for insomnia; ISI: Insomnia severity index 


\section{Acknowledgements}

We would like to thank: the patients at the clinic who participated in this research study; therapists Agnes Wirdby and Mikaela Grännby for acting as group leaders; department manager Holger Thomas and unit secretary Sigrid Ölund for their contribution and positive attitude towards our research at the clinic, and all other staff at the department of ADHD, Northern Stockholm Psychiatry, for fruitful collaboration.

\section{Authors' contributions}

SJ gave substantial contributions to the conception of the research and design of the study. SJ supervised YL and ML in the acquisition of data, analysed and interpreted the data, drafted the work and was responsible for and has read and approved the final version of the article. SJ agrees to be accountable for all aspects of the work in ensuring that questions related to the accuracy or integrity of any part of the work are appropriately investigated and resolved. YL gave substantial contributions to the conception of the research, acquisition and interpretation of data. $\mathrm{YL}$ participated in drafting the work and revising it, and has read and approved the final version of the article. YL agrees to be accountable for all aspects of the work in ensuring that questions related to the accuracy or integrity of any part of the work are appropriately investigated and resolved. ML gave substantial contributions to the conception of the research, acquisition and interpretation of data. ML participated in drafting the work and revising it, and has read and approved the final version of the article. ML agrees to be accountable for all aspects of the work in ensuring that questions related to the accuracy or integrity of any part of the work are appropriately investigated and resolved. BN gave substantial contributions to the interpretation of data. BN revised the work critically for important intellectual content, and has read and approved the final version of the article. BN agrees to be accountable for all aspects of the work in ensuring that questions related to the accuracy or integrity of any part of the work are appropriately investigated and resolved. VK gave substantial contributions to the interpretation of data. VK revised the work critically for important intellectual content, and has read and approved the final version of the article. VK agrees to be accountable for all aspects of the work in ensuring that questions related to the accuracy or integrity of any part of the work are appropriately investigated and resolved. All authors read and approved the final manuscript.

\section{Authors' information}

All authors are licensed clinical psychologists trained in CBT. $\mathrm{SJ}, \mathrm{PhD}, \mathrm{LP}$, principal investigator of this study, specialist in behavioral sleep medicine $Y L, M S c, L P M L, M S c, L P B K, P h D$, LP, specialist in ADHD VK, Professor, PhD, LP, research group leader

\section{Funding}

Not applicable (the project had no external funding).

\section{Availability of data and materials}

The datasets used and/or analyzed during the current study are available from the corresponding author on reasonable request.

\section{Ethics approval and consent to participate}

The study protocol was registered and approved by the regional ethical review board in Stockholm, Sweden on January 13th 2016 (Study id: 2015/2078-31/1), and written informed consent was obtained from all participants.

\section{Consent for publication}

Not applicable.

\section{Competing interests}

The authors declare that they have no competing interests.

\section{Author details}

'Division of Psychology, Department of Clinical Neuroscience, Karolinska Institutet, Stockholm, Sweden. ${ }^{2}$ Centre for Psychiatry Research, Department of Clinical Neuroscience, Karolinska Institutet, \& Stockholm Health Care Services, Stockholm County Council, Stockholm, Sweden. ${ }^{3}$ Department of Psychology, Faculty of Health and Life Sciences, Linnaeus University, Växjö, Sweden.
Received: 9 February 2019 Accepted: 17 July 2019

Published online: 24 July 2019

\section{References}

1. Grane VA, Endestad T, Pinto AF, Solbakk A-K. Attentional control and subjective executive function in treatment-naive adults with attention deficit hyperactivity disorder. PLoS One. 2015;9(12):e115227.

2. Simon V, Czobor P, Bálint S, Mészáros A, Bitter I. Prevalence and correlates of adult attention- deficit hyperactivity disorder: meta- analysis. $\mathrm{Br}$ J Psychiatry. 2009;194(3):204.

3. National Institute for Health and Care Excellence. Attention deficit hyperactivity disorder: diagnosis and management (NG87). London: NICE; 2018. Available from: https://www.nice.org.uk/guidance/ng87.

4. Safren SA, Otto MW, Sprich S, Winett CL, Wilens TE, Biederman J. Cognitivebehavioral therapy for ADHD in medication- treated adults with continued symptoms. Behav Res Ther. 2005;43(7):831-42.

5. Nasri B, Castenfors M, Fredlund P, Ginsberg Y, Lindefors N, Kaldo V. Group Treatment for Adults With ADHD Based on a Novel Combination of Cognitive and Dialectical Behavior Interventions. A Feasibility Study Journal of Attention Disorders. 2017;ahead of print:1-13.

6. Sobanski E. Psychiatric comorbidity in adults with attention-deficit/ hyperactivity disorder (ADHD). Eur Arch Psychiatry Clin Neurosci. 2006; 256(1):i26-31.

7. Hvolby A. Associations of sleep disturbance with ADHD: implications for treatment. Atten Defic Hyperact Disord. 2015;7(1):1-18.

8. Bjorvatn B, Brevik EJ, Lundervold AJ, Halmøy A, Posserud M-B, Instanes JT, et al. Adults with Attention Deficit Hyperactivity Disorder Report High Symptom Levels of Troubled Sleep, Restless Legs, and Cataplexy. Front Psychol. 2017;8:1621.

9. Schredl M, Alm B, Sobanski E. Sleep quality in adult patients with attention deficit hyperactivity disorder (ADHD). Eur Arch Psychiatry Clin Neurosci. 2007;257(3):164-8.

10. Wynchank D, Bijlenga D, Beekman AT, Kooij JJS, Penninx BW. Adult attention-deficit/hyperactivity disorder (ADHD) and insomnia: an update of the literature. Curr Psychiatry Rep. 2017;19(12):98.

11. Brevik EJ, Lundervold AJ, Halmøy A, Posserud MB, Instanes JT, Bjorvatn B, et al. Prevalence and clinical correlates of insomnia in adults with attentiondeficit hyperactivity disorder. Acta Psychiatr Scand. 2017;136(2):220-7.

12. Bijlenga D, Van Someren EJW, Gruber R, Bron TI, Kruithof IF, Spanbroek ECA, et al. Body temperature, activity and melatonin profiles in adults with attention- deficit/ hyperactivity disorder and delayed sleep: a case- control study. J Sleep Res. 2013;22(6):607-16.

13. Kooij J, Bijlenga D. The circadian rhythm in adult attention- deficit/ hyperactivity disorder: current state of affairs. Expert Rev Neurother. 2013;13: 1107-16.

14. Gamble KL, May RS, Besing RC, Tankersly AP, Fargason RE. Delayed sleep timing and symptoms in adults with attention-deficit/hyperactivity disorder: a controlled Actigraphy study. Chronobiol Int. 2013;30(4):598-606.

15. van Geijlswijk IM, Korzilius HPLM, Smits MG. The use of exogenous melatonin in delayed sleep phase disorder: a Meta-analysis. Sleep. 2010; 33(12):1605-14.

16. Bendz LM, Scates AC. Melatonin treatment for insomnia in pediatric patients with attention-deficit/hyperactivity disorder. Ann Pharmacother. 2010:44(1):185-91.

17. Rybak YE, McNeely H, Mackenzie B, Jain U, Levitan R. An open trial of light therapy in adult attention-deficit/hyperactivity disorder. J Clin Psychiatry. 2006;67(10):1527-35.

18. Fargason RE, Fobian AD, Hablitz LM, Paul JR, White BA, Cropsey KL, et al. Correcting delayed circadian phase with bright light therapy predicts improvement in ADHD symptoms: a pilot study. J Psychiatr Res. 2017; 91:105-10.

19. Pillai V, Roth T, Roehrs T, Moss K, Peterson EL, Drake CL. Effectiveness of Benzodiazepine Receptor Agonists in the Treatment of Insomnia: An Examination of Response and Remission Rates. Sleep. 2017;40(2):zsw044-zsw.

20. Blumer JL, Findling RL, Shih WJ, Soubrane C, Reed MD. Controlled clinical trial of zolpidem for the treatment of insomnia associated with attentiondeficit/ hyperactivity disorder in children 6 to 17 years of age. Pediatrics. 2009;123(5):e770-e6.

21. Morin CM. Insomnia: psychological assessment and management. New York: The Guilford Press; 1993. 238, 
22. Jernelöv S, Lekander M, Blom K, Rydh S, Ljótsson B, Axelsson J, et al. Efficacy of a behavioral self-help treatment with or without therapist guidance for co-morbid and primary insomnia - a randomized controlled trial. BMC Psychiatry. 2012;12:5.

23. Blom K, Jernelöv S, Kraepelien M, Olséni Bergdahl M, Jungmarker K, Ankartjärn $L$, et al. Internet treatment addressing either insomnia or depression, for patients with both diagnoses-a randomized trial. Sleep. 2015; 38(2):267-77.

24. National Institutes of Health. National Institutes of Health State of the Science Conference statement on manifestations and management of chronic insomnia in adults, June 13-15, 2005. Sleep. 2005;28:1049-57.

25. Schutte-Rodin SL, Broch L, Buysse D, Dorsey C, Sateia M. Clinical guideline for the evaluation and management of chronic insomnia in adults. J Clin Sleep Med. 2008:4(5):487-504.

26. Qaseem A, Kansagara D, Forciea MA, Cooke M, Denberg TD, Clinical Guidelines Committee of the American College of Physicians. Management of Chronic Insomnia Disorder in adults: a clinical practice guideline from the American College of Physicians. Ann Intern Med. 2016;165(2):125-33. https://doi.org/10.7326/M15-2175 Epub 16 May 3.

27. Riemann D, Baglioni C, Basetti C, Bjorvatn B, Dolenc Groselj L, Ellis JG, et al. European guideline for the diagnosis and treatment of insomnia. J Sleep Res. 2017;26:675-700.

28. Geiger-Brown J, Rogers VE, Liu W, Ludeman E, Downton K, Diaz-Abad M. Cognitive behavioral therapy in persons with comorbid insomnia: a Meta-analysis. Sleep Med Rev. 2015:23:54-67.

29. van Straten A, van der Zweerde T, Kleiboer A, Cuijpers P, Morin CM, Lancee J. Cognitive and behavioral therapies in the treatment of insomnia: a meta-analysis. Sleep Med Rev. 2018;38:3-16.

30. Freeman D, Sheaves B, Goodwin GM, Yu L-M, Nickless A, Harrison PJ, et al. The effects of improving sleep on mental health (OASIS): a randomised controlled trial with mediation analysis. Lancet Psychiatry. 2017:4(10):749-58.

31. Freeman D, Waite F, Startup H, Myers E, Lister R, Mclnerney J, et al. Efficacy of cognitive behavioural therapy for sleep improvement in patients with persistent delusions and hallucinations (BEST): a prospective, assessor-blind, randomised controlled pilot trial. Lancet Psychiatry. 2015;2(11):975-83.

32. Spoormaker VI, Verbeek I, van den Bout J, Klip EC. Initial validation of the SLEEP-50 questionnaire. Behav Sleep Med. 2005;3(4):227-46.

33. Ingre M, Van Leeuwen W, Klemets T, Ullvetter C, Hough S, Kecklund G, et al. Validating and extending the three process model of alertness in airline operations. PLoS One. 2014;9(10):e108679.

34. Bastien $\mathrm{CH}$, Vallières $\mathrm{A}$, Morin $\mathrm{CM}$. Validation of the insomnia severity index as an outcome measure for insomnia research. Sleep Med. 2001 2(4):297-307.

35. Kanstrup M, Holmström L, Ringström R, Wicksell RK. Insomnia in paediatric chronic pain and its impact on depression and functional disability. Eur J Pain. 2014;18:1094-102.

36. Adler LA, Spencer T, Faraone SV, Kessler RC, Howes MJ, Biederman J, et al. Validity of pilot adult ADHD self-report scale (ASRS) to rate adult ADHD symptoms. Ann Clin Psychiatry. 2006;18(3):145-8.

37. Safren SA, Perlman CA, Sprich S, Otto MW. Mastering your adult ADHD: a cognitivebehavioral treatment program - therapist quide: Oxford University Press; 2005.

38. Wu JQ, Appleman ER, Salazar RD, Ong JC. Cognitive behavioral therapy for insomnia comorbid with psychiatric and medical conditions: a metaanalysis. JAMA Intern Med. 2015;175(9):1461-72.

39. Jansson-Fröjmark M, Norell-Clarke A. Cognitive Behavioural therapy for insomnia in psychiatric disorders. Curr Sleep Med Rep. 2016;2(4):233-40.

40. Huisman M. Imputation of missing item responses: some simple techniques. Qual Quant. 2000:34(4):331-51.

41. Borenstein M, Hedges LV, Higgins JPT, Rothstein HR. Introduction to metaanalysis. Chichester: Wiley; 2009.

42. Rubin DB, Schenker N. Multiple imputation in health-are databases: an overview and some applications. Stat Med. 1991;10(4):585-98.

43. Barnard J, Rubin DB. Miscellanea. Small-sample degrees of freedom with multiple imputation. Biometrika. 1999:86(4):948-55.

44. Harvey AG. A Transdiagnostic intervention for youth sleep and circadian problems. Cogn Behav Pract. 2016;23(3):341-55.

45. Harvey AG, Buysse DJ. Treating sleep problems: a transdiagnostic approach. New York: The Guilford Press; 2017

46. Sheaves B, Isham L, Bradley J, Espie C, Barrera A, Waite F, et al. Adapted CBT to Stabilize Sleep on Psychiatric Wards: a Transdiagnostic Treatment Approach. Behav Cogn Psychother. 2018;46(6):661-75.
47. Sheaves B, Freeman D, Isham L, Mclnerney J, Nickless A, Yu L-M, et al. Stabilising sleep for patients admitted at acute crisis to a psychiatric hospital (OWLS): an assessor-blind pilot randomised controlled trial. Psychol Med. 2018:48(10):1694-704.

48. Harvey AG. Sleep and circadian rhythms in bipolar disorder: seeking synchrony, harmony, and regulation. Am J Psychiatr. 2008;165(7):820-9.

49. Wulff K, Dijk D-J, Middleton B, Foster RG, Joyce EM. Sleep and circadian rhythm disruption in schizophrenia. Br J Psychiatry. 2012;200(4):308-16.

50. Spielman AJ, Caruso LS, Glovinsky PB. A behavioral perspective on insomnia treatment. Psychiatr Clin N Am. 1987;10(4):541-53.

51. Bonnet $\mathrm{MH}$, Arand DL. Hyperarousal and insomnia: state of the science. Sleep Med Rev. 2010;14(1):9-15.

52. Weibel S, Jermann F, Weiner L, Nicastro R, Ardu S, Pham E, et al. Insomnia in adult attention-deficit/hyperactivity disorder: a comparison with borderline personality disorder population in a clinical setting and control participants. Compr Psychiatry. 2017;76:119-28.

\section{Publisher's Note}

Springer Nature remains neutral with regard to jurisdictional claims in published maps and institutional affiliations.
Ready to submit your research? Choose BMC and benefit from:

- fast, convenient online submission

- thorough peer review by experienced researchers in your field

- rapid publication on acceptance

- support for research data, including large and complex data types

- gold Open Access which fosters wider collaboration and increased citations

- maximum visibility for your research: over $100 \mathrm{M}$ website views per year

At $\mathrm{BMC}$, research is always in progress.

Learn more biomedcentral.com/submissions 